\title{
BIM Project Execution Plan for Infrastructure Superintendence (SINFRA) of the Federal University of Pernambuco: presentation of a case study
}

\author{
Isabella Rodrigues Oliveira da Silva \\ UFPE | Brasil | isabellarodrigues097@gmail.com
}

Max Lira Veras Xavier de Andrade

UFPE | Brasil | max.andrade@ufpe.br

\begin{abstract}
This paper shows the importance of implementing a BIM Execution Plan (BEP) as an instrument for the successfully BIM diffusion. BEP specifies the scope required for implementation, identifies processes, defines exchanges of information and describes needs of the project to provide adequate support for BIM implementation. The proposed BEP is to be implemented in the Design Sector (SINFRA) of a Brazilian Public University. It started with a diagnosis, choosing a pilot design project, defining the team to propose a BEP. This work presents the partial result of this BEP implementation, showing the benefits and the importance for the SINFRA.
\end{abstract}

Keywords: Building Information Modeling (BIM); BIM Execution Plan (BEP); BIM implementation in public company; Design Process with BIM.

\section{INTRODUÇÃO}

O atual cenário brasileiro ainda é carente quando se trata da implementação do BIM nos escritórios de projeto e construtoras. As práticas BIM são pouco difundidas, e, muitas vezes, mal interpretadas, o que tem resultado em diversos casos de insucesso na sua execução. Isso acontece, principalmente, pela falta de conhecimento dos gerentes e diretores de projetos sobre as reais necessidades que estão vinculadas às práticas BIM no projeto (Leusin, 2018). É muito comum o desconhecimento em como se dá a adoção do BIM. Muitas empresas renegam a compreender que as práticas vinculadas ao BIM resultam em novos modelos contratuais, novas posturas durante as práticas de projeto e execução de obra e novos modelos de colaboração e comunicação. É muito comum, ainda, o BIM estar associado ao uso de ferramentas de modelagem baseada em objetos, as conhecidas Plataformas BIM (Revit, Archicad, OpenBuidling, entre outros softwares).

Por outro lado, diversas iniciativas nacionais têm estimulado a adoção do BIM no cenário Brasileiro. Entre as diversas ações, destaca-se o Decreto $n^{0} 10.306$, de 2 de abril de 2020, que "estabelece a utilização do Building Information Modelling na execução direta ou indireta de obras e serviços de engenharia realizada pelos órgãos e pelas entidades da administração pública federal, no âmbito da Estratégia Nacional de Disseminação do Building Information Modelling - Estratégia BIM BR, instituída pelo Decreto $n^{\circ}$ 9.983, de 22 de agosto de 2019". Esta estratégia é apoiada por diversos indicadores que visam direcionar o processo de implementação ao longo de 10 anos em empreendimentos públicos federais brasileiros. A proposta defendida é que o BIM seja incorporado em todas as fases do ciclo de vida do empreendimento. Inicia-se pela fase de projeto, a segunda fase envolve o projeto e construção e a última fase inclui o projeto, a construção e o uso e manutenção das construções públicas federais, novas e as preexistentes.

A partir da necessidade de utilização do BIM expressa pelo decreto citado acima, diversas empresas públicas têm investido recursos visando a implementação deste em suas instituições. Muitas delas ainda estão em estágio inicial. Outras, como o Exército Brasileiro, através do Sistema Unificado do Processo de Obras (OPUS), já desenvolveram programas pilotos visando auxiliar na construção e manutenção da infraestrutura edificada dessa instituição.

É dentro desse contexto que o presente trabalho se insere. Este foi desenvolvido como parte de um Trabalho de Conclusão do Curso de Graduação em Arquitetura e Urbanismo (ainda em desenvolvimento), cujo objetivo é auxiliar a Superintendência de Infraestrutura (SINFRA) da Universidade Federal de Pernambuco (UFPE) na introdução do BIM, por meio da criação de um Plano de Execução BIM (BIM Execution Planning - BEP). A SINFRA é um órgão da UFPE cuja finalidade é o planejamento e administração das obras edificadas e do uso dos espaços físicos dessa universidade. $\mathrm{O}$ trabalho a ser apresentado nesse artigo visa discutir a 
implementação do BEP na SINFRA, indicando os estágios já implementados e os desafios a serem alcançados.

\section{OBJETIVO}

O objetivo desse artigo, portanto, é apresentar os estágios usados para a construção de um Plano de Execução BIM (BEP) cuja ênfase se deu no desenvolvimento de um anteprojeto de arquitetura e complementares para a construção de um anexo a um edifício existente no campus da UFPE. Projetos esses realizados pela SINFRA.

Como objetivos específicos deste artigo estão:

- Conceituar o BEP e descrever as etapas necessárias para a sua realização;

- Criar a estrutura geral de um BEP para a realização de anteprojetos de arquitetura e de projetos complementares junto à SINFRA;

- Descrever os fluxos de projeto, os modelos de colaboração e os tipos de entregáveis necessários para a criação do BEP apresentado neste artigo.

\section{METODOLOGIA}

O método proposto para o desenvolvimento do BEP foi o Design Science Research (DSR). Este constitui-se em um método para a resolução de problemas de projeto de ordem teórico e prático. O DSR visa criar e avaliar um artefato que permita a resolução satisfatória para um problema prático (Lacerda, 2013). O Artefato no caso desse trabalho é o BEP. Para a estruturação deste BEP a presente pesquisa se baseou no Project Execution Planning Guide, Version 2.2 (Messner et al., 2019). A partir das fases propostas por esse guia, a criação do BEP foi delineada. Após a criação do artefato, a fase seguinte - ainda a ser executada - constituirá na avaliação dos benefícios para a SINFRA da aplicação do BEP no projeto estudado.

Como primeiro estágio para a constituição do artefato foi realizado uma fase de imersão no problema do artefato. Esta se deu por meio de uma pesquisa bibliográfica em algumas das principais publicações, brasileiras e internacionais em BEP. Entre os documentos pesquisados destacam-se Messner et al., (2019) e Leusin (2018).

\section{REVISÃO DE LITERATURA BIM}

Para Eastman et al. (2014), o BIM não é uma coisa ou um tipo de software, mas uma atividade humana que envolve mudanças amplas no processo de construção. Para Succar (2009), o BIM constitui-se de um conjunto de políticas, processos e tecnologias que geral uma metodologia para gerenciar o projeto e os dados do edifício de forma digital, por todo o ciclo de vida do edifício. O BIM envolve a gestão da informação da construção por meio de ações colaborativas que proporcionam uma base para as novas capacidades da construção. Isso, associado aos sistemas e dados inovadores, contribui para o desenvolvimento das equipes envolvidas no empreendimento.

\section{BEP}

O Plano de Execução BIM (BEP) consiste em um manual que estabelece as diretrizes para a implementação do BIM nas empresas da indústria de Arquitetura, Engenharia, Construção e Operação (AECO), de forma planejada e escalonada, a fim de alcançar os objetivos definidos. O BEP deve especificar todo o escopo necessário à implementação, identificar os processos de cada atividade, definir as trocas de informação entre as partes interessadas e descrever as necessidades do projeto e infraestrutura essenciais para dar o suporte adequado ao processo de implementação.

É importante, ainda, que o BEP especifique os responsáveis por executar cada um dos processos e atividades e os seus prazos de entrega para que tenha um maior controle de todo o processo do projeto, da construção, do planejamento até a manutenção do empreendimento. O objetivo do BEP deve ser, portanto, efetivamente integrar o BIM em todo o processo das entregas do projeto, implementando-o a um nível que maximize o seu valor ao passo que diminua os seus custos e os seus impactos, o que pode garantir uma maior eficiência na implementação do BIM.

As etapas do BEP, descritas a seguir foram guiadas pelo "BIM Project Execution Planning Guide, Version 2.2" que foi produzido pela Pennsylvania State University em 2019 (Messner et al., 2019) e adaptado para o presente trabalho de modo que se adeque a realidade da SINFRA. Tendo dito isso, faz-se necessária a explicação dos conceitos base que serão utilizados ao longo de todo o Plano de Execução BIM (BEP).

O BEP se inicia com o diagnóstico da empresa e do projeto ao qual se pretende iniciar o BIM (Projeto Piloto). Nessa fase devem ser identificados: o proprietário ou responsável do projeto; o nome do projeto; o endereço; uma breve descrição do projeto; o diagnóstico atual do processo de projeto; o número de disciplinas a serem desenvolvidas, o cronograma e as fases do projeto. Essas informações servem para construção de um diagnóstico inicial da empresa no qual o BEP manager utilizará como base para identificar o perfil da empresa e do projeto.

Após isso, é importante especificar todas as entregas que serão feitas, as fases do projeto, as disciplinas de projeto e seus respectivos documentos. Essas informações devem ser construídas para um projeto específico, não devendo serem aplicadas a todos os projetos de uma empresa.

O próximo passo constitui-se em definir os objetivos BIM, que servem para determinar os interesses da organização para com o BIM. Para identificar esses objetivos, devem-se levar em consideração as características particulares do projeto, as habilidades do time e os riscos das atividades. Os objetivos BIM precisam estar em total consonância com o planejamento estratégico da empresa para que sejam implementados de acordo com os planos futuros da organização. Os objetivos escolhidos precisam ser mensuráveis e alinhados a melhoria dos processos de planejamento, projeto, construção e operação da empresa (Messner et al., 2019). 
Depois de alinhar os objetivos BIM, que são gerais à organização, é preciso definir os objetivos do projeto. Esses objetivos são referentes ao projeto específico ao qual o BEP será aplicado. Devem considerar a finalidade daquele projeto e o que deve ser entregue na conclusão dele.

Após definição dos objetivos e suas metas, é importante traduzi-los para os Usos BIM respectivos aos interesses da organização, a fim de destrinchá-los ao longo do BEP. Estes têm caraterísticas e especificidades que guiarão toda a implementação do BIM e devem ser definidos junto a gestão estratégica da empresa. São especificidades dos Usos BIM: identificação dos potenciais Usos BIM; identificação das partes responsáveis para cada Uso BIM; avaliação das capacidades necessárias aos responsáveis para cada Uso BIM (recursos, competência e experiência); e determinação de quando implementar cada Uso BIM.

Após esse processo inicial de diagnóstico e definição dos Usos BIM, é analisado e proposto um novo fluxo de processos a ser implementado na empresa. Todas essas informações ficam registradas e farão parte do BEP. Este se constitui em um documento base para a formação dos contratos de projetos baseados em uma prática BIM.

É a partir desse roteiro proposto por Messner et al. (2019) que a implementação do BEP junto à SINFRA está sendo conduzida. A seguir, serão apresentadas as etapas que já foram realizadas para implementação do BEP e os desafios que ainda estão por vir.

\section{RESULTADOS}

Como ponto de partida para a implementação do BIM na SINFRA, o trabalho apresentado nesse artigo criou um BEP para o desenvolvimento de um anteprojeto de arquitetura e complementares. Neste, estão sendo destrinchadas as ações para o desenvolvimento dos anteprojetos (arquitetura e complementares) considerando os fluxos, as entradas de dados e modelos de colaboração e gestão, a serem utilizados pela SINFRA, para guiar a implementação do BIM. O BEP está sendo construído de forma colaborativa com a equipe de projetos a fim de garantir a aplicabilidade, a partir do engajamento dos colaboradores com a solução da proposta.
Embora ainda em desenvolvimento, os resultados iniciais da pesquisa demonstram a importância, dentro de um processo de implementação BIM, da criação do BEP. Mostra também que a implementação do BIM pode ser destrinchada em vários estágios, dentro de um cronograma de curto, médio e longo prazo. Para isso, é recomendável a criação de vários BEPs. Dessa forma, é possível estabelecer um processo de implementação com uma evolução mais consistente, apoiada na realidade da empresa naquele momento. O primeiro passo para a implementação do BIM/BEP foi a realização de um diagnóstico na SINFRA. Com a realização do diagnóstico foi confirmada a necessidade de um processo de reestruturação do setor, alinhado ao planejamento estratégico da instituição.

\section{DIAGNÓSTICO}

A SINFRA é constituída por cinco diretorias (Administrativa, Gestão Ambiental, Manutenção e Conservação, Planos e Projeto e Fiscalização de Obra), cada uma das quais com objetivos e funções diversas, mas interligadas. Para o estágio inicial de implementação do BIM foi escolhida a Diretoria de Planos e Projetos. Esta é responsável pelo desenvolvimento de projetos (arquitetura e complementares) e orçamentação. O diagnóstico realizado na Diretoria de Planos e Projetos foi baseado nas informações cedidas pela diretora desse órgão em reunião ocorrida em outubro de 2019. O setor de Projetos é composto por 22 pessoas, sendo 10 deles estagiários remunerados. $\mathrm{O}$ contato com os concursos de arquitetura para inserção de novas pessoas na equipe é inexistente, havendo uma defasagem entre as demandas humanas do setor de projetos e os concursados aprovados. Os editais, portanto, não são abertos para os cargos e especialidades em vacâncias da equipe, o que sobrecarrega algumas disciplinas de projeto.

Com base nisso, a SINFRA necessita fazer um programa de capacitação a cada contratação de novos funcionários para que estes possam ser redirecionados para alguma área mais necessitada do setor, não aproveitando totalmente as capacidades dos novos colaboradores.

A subcontratação de projetos é decidida de acordo com a necessidade de cada projeto. É baseada nas disciplinas que a empresa não tem especialista, como demonstra o fluxo de projetos da Figura 1.

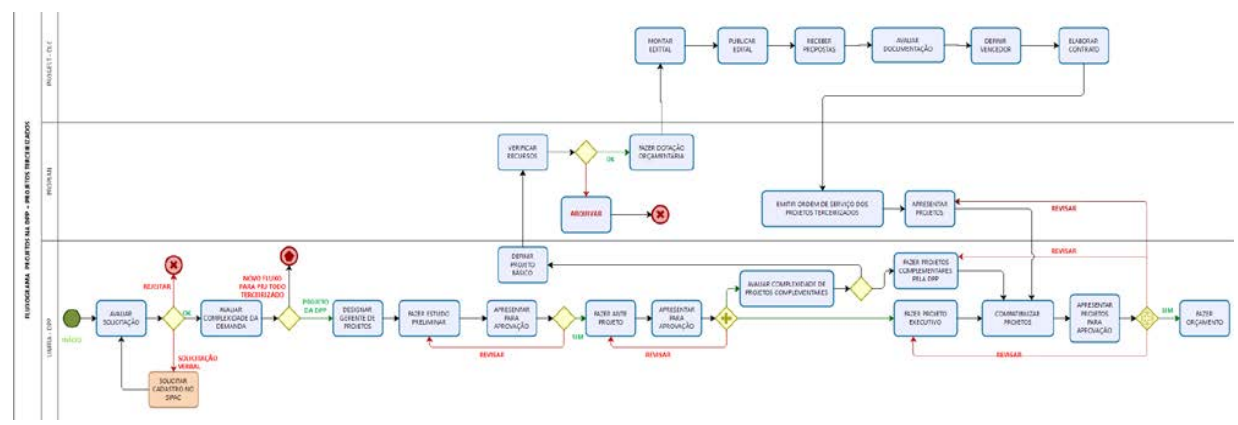

Figura 1: Fluxograma de projetos atual da SINFRA. Fonte: produção interna da SINFRA. 
A SINFRA executa projetos de complexidade bem variada, desde simples manutenções de revestimento e pintura a grandes centros educacionais dentro do campus da universidade. Essa variação na escala dos projetos faz com que seja necessário, ao receber uma demanda por parte de um cliente - que pode ser a Reitoria ou os Centros - avaliar a complexidade do pedido, a fim de direcionar os esforços para a equipe interna e/ou contratar um terceirizado. Caso seja necessário a contratação de um projeto terceirizado, é preciso que este passe por um processo licitatório estruturado com a publicação de edital e escolha do projetista vencedor, conforme mapeado na Figura 1.

Para aplicar do BEP no setor foi escolhido um projeto piloto. O projeto consiste em uma ampliação no edifício do Departamento de Física da UFPE, com a construção de um pequeno bloco de banheiros acessíveis. Para essa obra será necessário projetos de arquitetura, hidrossanitário, elétrico, estrutura além da orçamentação. Pelo porte do projeto, foi classificado como de baixa complexidade, sendo todos os projetos realizados pela equipe interna da empresa. A equipe destinada aos projetos é composta por 02 arquitetos, 01 engenheiro eletricista, 01 engenheiro hidrossanitário, 01 engenheiro orçamentista e 01 engenheiro calculista.

\section{OBJETIVOS DO PROJETO}

Com base nas entrevistas de diagnóstico, foi possível definir os interesses da empresa para com o BIM, a fim de alavancar seus processos internos. Esta tem como objetivos essenciais: (1) melhor colaboração entre as equipes e as disciplinas de projeto; (2) compatibilização dos projetos com a identificação de possíveis conflitos entre disciplinas; (3) melhoria nas estimativas de custo do empreendimento com uma orçamentação mais precisa.

Ao analisar o fluxo de projetos atual, percebe-se que o orçamento é construído após a avaliação da demanda, com base em uma especificação básica de projeto, e ao final, após a aprovação do projeto executivo. Dessa forma, entre a estimativa de custo inicial e o orçamento final pode existir uma diferença grande, em termos financeiros, pois a estimativa de custo apenas usa como base o conceito do projeto e a tabela de referência de preços padrão da empresa.

No que se refere à colaboração, as equipes de projetos são montadas com dois arquitetos e um projetista para cada disciplina complementar. Como são profissionais do quadro da SINFRA em teoria poderia ser um ponto positivo para a existência de uma colaboração efetiva. Todavia, na prática a colaboração entre as disciplinas de projeto não existe, ou quase não existe. Os projetos complementares e de estrutura apenas entram no fluxo de arquitetura após a fase de projeto executivo, sendo que, em muitos casos, não há qualquer compatibilização ou revisão. Estas acontecem apenas quando há exigência pela equipe de orçamentação. Esse processo tardio, para não dizer inexistente, de checagem de interferências faz com que muitas informações cruciais não sejam discutidas no estudo preliminar nem no anteprojeto, e, quando ocorre, apenas na etapa de projeto executivo, causando retrabalhos e podendo influenciar no custo final do projeto ou da obra, visto que aquele se encontra em fase final de detalhamento para entrega do projeto para a licitação e posterior construção.

\section{USOS BIM}

A definição dos usos do Modelo BIM se dá por meio dos desdobramentos dos objetivos da empresa para com o BIM. A partir da definição desses objetivos, são detalhados os usos do Modelo BIM e especificados quais as ações necessárias para se chegar a cada um dos usos requeridos pela empresa.

A Tabela 1 apresenta os objetivos iniciais da SINFRA para com o BIM e seus respectivos usos BIM visando a implementação de um primeiro projeto piloto, já especificado acima.

Tabela 1: Usos BIM associados aos objetivos do projeto.

\begin{tabular}{|c|l|l|}
\hline $\begin{array}{c}\text { IMPORTÂNCIA } \\
\text { (alta, média, } \\
\text { baixa) }\end{array}$ & $\begin{array}{c}\text { OBJETIVOS DO } \\
\text { PROJETO }\end{array}$ & \multicolumn{1}{c|}{$\begin{array}{c}\text { USOS BIM } \\
\text { ASSOCIADOS }\end{array}$} \\
\hline ALTA & $\begin{array}{l}\text { Melhoria na } \\
\text { estimativa de custo } \\
\text { e na qualidade da } \\
\text { orçamentação para } \\
\text { obra }\end{array}$ & $\begin{array}{l}\text { Estimativa de } \\
\text { custo }\end{array}$ \\
\hline ALTA & $\begin{array}{l}\text { Melhoria na } \\
\text { compatibilização } \\
\text { dos projetos }\end{array}$ & $\begin{array}{l}\text { Detecção de } \\
\text { conflitos }\end{array}$ \\
\hline MÉDIA & $\begin{array}{l}\text { Maior colaboração } \\
\text { entre a equipe e as } \\
\text { disciplinas de } \\
\text { projeto }\end{array}$ & Projeto 3D \\
\hline \multicolumn{2}{|c|}{ Fonte: Autores baseado em Succar (2018). } \\
\hline
\end{tabular}

No projeto piloto, optou-se por destrinchar os fluxos de projeto considerando os Usos do Modelo BIM definidos na Tabela 1.

\section{Modelo Arquitetônico:}

Esse modelo deve ser utilizado a fim de explorar as opções de projeto e servir como base para o modelo federado, ou seja, o modelo que consta com todas as disciplinas associadas e finalizadas. Esse modelo é, portanto, utilizado para integração das disciplinas e deve suportar a coordenação de projeto com o "clash detection" e as revisões do modelo. $\mathrm{O}$ time de arquitetura fica responsável pelo desenvolvimento do modelo base de arquitetura e deve definir as coordenadas para todas as disciplinas complementares. O modelo deve ser projetado no LOD (Nível de Desenvolvimento) 300, com a especificação de: elementos de formas genéricas e aproximadas das suas dimensões, pesos, quantidades, orientações e localizações. Informações não geométricas podem ser anexadas ao modelo (Leusin, 2018).

\section{Modelos de Estrutura, de instalações hidrossanitárias e de instalações elétricas}

Os modelos de estrutura, de instalações hidrossanitárias e de instalações elétricas ocorrem como projetos complementares ao projeto arquitetônico. O modelo de estrutura deve fornecer informações sobre as geometrias da estrutura (pilares, vigas, lajes, fundações e escadas) e 


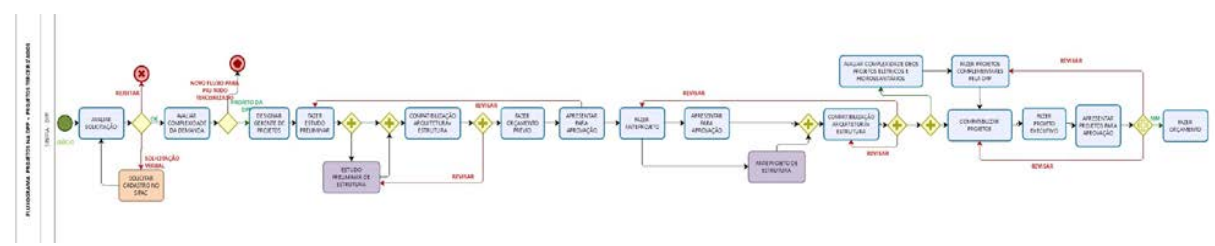

Figura 2: Fluxograma de projetos proposto até o Anteprojeto - SINFRA (revisão 00). Fonte: Autores.

quantificativos de ferragens, escavações e fôrmas. Os modelos de instalações hidrossanitárias e de instalações elétricas devem conter os componentes corretamente especificados com suas dimensões e desenhos além de ter uma codificação conforme o Sistema Nacional de Pesquisa de Custos e Índices da Construção Civil (SINAPI).

Uso dos Modelos BIM para Estimativa de custos visando a orçamentação

A criação de estimativas de custo se dará com base nos espaços modelados em software de autoria BIM. Estas poderão ser facilmente calculadas com base no tipo de construção e nas áreas e volumes gerados pela equipe de projeto. A SINFRA já vem nos últimos anos tentando criar um índice para estimar os custos de diferentes tipos de espaço e de projetos desenvolvidos no campus universitário, mas com a importação de padrões de construção e acabamentos nos Templates BIM, acreditase que isso será bem mais fácil, com a possibilidade de realização de uma estimativa de custo razoavelmente próxima do real, ainda no estudo preliminar.

Para auxiliar a orçamentação, propõe-se extrair os quantitativos do modelo, já compatibilizado, com um LOD de 300 . Esse modelo terá suas saídas configuradas para extrair os quantitativos conforme o método de cálculo usado para orçamentação na SINFRA, que se dá com o uso de tabelas de referências no Excel e usando a codificação da SINAPI

\section{FLUXOGRAMA DE PROJETO PROPOSTO NO BEP}

Com base nos objetivos do projeto, na intenção da instituição para com o BIM e nos usos BIM, fol remodelado o fluxograma do setor de projetos. Para este BEP a proposta se deu para a implementação do BIM da etapa de concepção à finalização dos anteprojetos de arquitetura e complementares. A definição do escopo do BEP também se deu em função do período destinado ao trabalho de conclusão de Curso (de 1 ano) e das especificidades necessárias a um BEP. Nesse contexto, os fluxogramas a seguir abrangem desde a chegada da demanda até a finalização do anteprojeto de arquitetura e complementares.

A proposta do novo fluxograma (Figura 2) foi de antecipar a entrada dos projetos de estrutura e de instalações para a etapa de anteprojeto de arquitetura. Tal decisão se deu a partir das premissas do BIM, o qual deve ser previstos os possíveis erros de projetos das diversas disciplinas e ajustados a fim de ter um modelo virtual semelhante ao executado na obra. Isso auxilia a mitigar o problema recorrente na SINFRA de problemas corriqueiros de colaboração e comunicação entre as disciplinas.

Sendo assim, tem-se na etapa de estudo preliminar de arquitetura o envio do projeto para o engenheiro de estrutura e o recebimento do estudo preliminar de estrutura para ser compatibilizado com o de arquitetura (Figura 2). O estudo preliminar de estrutura trata-se de um pré-dimensionamento e escolha do sistema construtivo, a fim de auxiliar o projeto de arquitetura e o orçamento. Após a compatibilização, são realizados os ajustes necessários em cada projeto e é avançado para a realização de estimativa de custo do empreendimento, com base nesse modelo já revisado. Para finalizar essa etapa, o estudo preliminar é apresentado para aprovação e, caso aceito, passa-se a fase de anteprojeto de arquitetura.

$\mathrm{Na}$ fase de anteprojeto de arquitetura é realizado um novo envio para estrutura e compatibilizado com o anteprojeto de estrutura recebido. Tendo feito a revisão com base na compatibilização, o projeto de arquitetura é enviado para a execução dos anteprojetos complementares de elétrica e hidrossanitário. É feita uma nova etapa de compatibilização com os anteprojetos de arquitetura, estrutura e complementares a fim de eliminar as inconsistências projetuais. Existe novamente uma etapa de apresentação dos projetos para aprovação e, então, os anteprojetos são enviados para a realização do projeto executivo, com a criação das respectivas documentações (plantas, cortes, fachadas, etc.).

Vale reiterar, ainda, que todo o processo de concepção do fluxograma foi feito em consonância com a diretoria de projetos, bem como a equipe referente ao projeto piloto escolhido, por meio de reuniões virtuais de apresentação e discussão. Tais reuniões geraram a revisão 01 presente neste artigo (Figura 3).

Após discussões com cada um dos projetistas da equipe e seus entregáveis, foi-se inteirado que dois engenheiros complementares desenvolvem seus projetos desenhando-os na mão, o que dificultaria o processo de compatibilização proposto no primeiro fluxograma (Figura 2). Dessa forma, foi proposta a revisão 01 do fluxograma (Figura 3), no qual foi acrescentada a etapa de modelagem dos anteprojetos complementares em um software de autoria BIM por um estagiário e aprovado pelo engenheiro sênior, adaptando-se a necessidade expressa pela equipe. É válido pontuar, ainda, que não é porque existem etapas que não são concebidas em softwares de autoria BIM que invalida a metodologia e a aplicação do BEP (Sacks et al., 2018). 


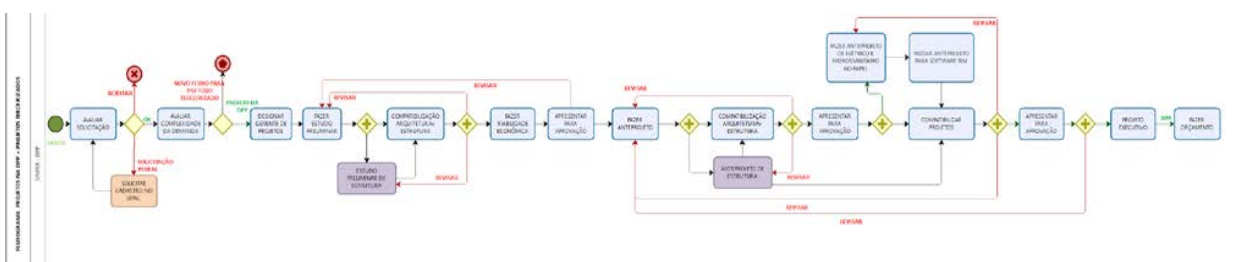

Figura 3: Fluxograma de projetos proposto até o Anteprojeto - SINFRA (revisão 01). Fonte: Autores.

\section{CONSIDERAÇÕES FINAIS}

O desenvolvimento do trabalho tem como cerne a demanda pela utilização do BIM para melhoria da gestão das informações e aumento do trabalho colaborativo, especialmente dentro da SINFRA. Além da Estratégia BIM BR citada anteriormente, o Governo ainda tem como meta o aumento em 10 vezes sobre a implantação do BIM no Brasil, fazendo com que $50 \%$ do PIB da construção civil tenha adotado o BIM até 2024, quando hoje apenas $9,2 \%$ adota, de acordo com a pesquisa da Fundação Getúlio Vargas (FGV IBRE). Isso confirma a necessidade de investimento em implementação adequada de metodologias que deem suporte ao BIM, de modo a garantir bons resultados na sua utilização.

A proposta de desenvolver um BEP e sua aplicação permitiu o aprofundamento do conhecimento teórico, além da aplicação desse conhecimento em uma prática de projeto já consolidada, ativa e que apresenta uma série de deficiências de gestão e de práticas colaborativas.

Durante o processo de implementação do BEP, ao buscar a sensibilização dos funcionários do setor de projetos da SINFRA quanto à importância da adoção do BIM e à necessidade de mudanças estruturais para sua adequada implantação, a pesquisa deparou com a necessidade de alterações nos fluxos propostos, adaptando-os à realidade local. Isso permitiu melhorar a qualidade do $\mathrm{BEP}$, principalmente no que se refere à adaptabilidade das capacidades técnicas dos funcionários e ao perfil do fluxo a ser implementado.

A conscientização da direção da SINFRA e dos funcionários da Diretoria de Planos e Projetos foi outro grande desafio. Mostrar que a adoção do BIM passa não apenas pelo uso de ferramentas e plataformas BIM, mas por uma mudança na forma como os projetistas se relacionam e trocam informações foi também outro grande desafio. Mais que simplesmente usar novas ferramentas, a proposta passou por conscientizar a equipe na necessidade de mudar a cultura de projeto, transformando os processos de comunicação e colaboração. Este foi e ainda continuam a ser um grande desafio para a Implementação do BIM e especificamente do BEP proposto e parcialmente apresentado neste artigo. A etapa seguinte, a ser implementada a partir dos próximos meses é de avaliar a qualidade do BEP proposto, identificado o que funcionou e o que precisa melhorar. Mas, essa etapa de avaliação do BEP fica para outra publicação.

\section{REFERÊNCIAS}

Brasil. Decreto $n^{\circ} \mathrm{N}^{0} 10.306$, de 2 de abril de 2020. Estabelece a utilização do Building Information Modelling - BIM ou Modelagem da Informação da Construção na execução direta ou indireta de obras e serviços de engenharia. Diário Oficial da União. Disponível em: http://www.planalto.gov.br/ccivil 03/ ato20192022/2020/decreto/D10306.htm

Delatorre, V. Modelo conceitual que integra BIM nas fases iniciais do projeto arquitetônico: uma abordagem para o processo criativo e de ensino-aprendizagem. Florianópolis/SC. 2019. Disponivel em: \&lt;https://core.ac.uk/download/pdf/30405331.pdf\&gt;.

Eastman, C. (2014) et al. Manual de BIM: um guia de modelagem da informação da construção para arquitetos, engenheiros, gerentes, construtores e incorporadores. Tradução: Cervantes Gonçalves Ayres Filho et al. Porto Alegre: Bookman.

Lacerda, D. (2013). Design Science Research: método de pesquisa para a engenharia de produção (pp. 741-761). Gest. Prod., São Carlos.

Leusin, S. (2018). Gerenciamento e Coordenação de Projetos BIM: Um guia de ferramentas e boas práticas para o sucesso de empreendimentos. 1. ed. Rio de Janeiro: Elsevier. ISBN 9788535290042 .

Messner, J. et al. (2019). BIM Project Execution Planning Guide, Version 2.2. Computer Integrated Construction Research Program, The Pennsylvania State University, University Park, PA, USA, August, Disponível em: http://bim.psu.edu.

Robalo, A.; Silva, F.; Rodrigues, I.; Lança, L.; Chalaça, P. (2015). BIM Project Execution Plan: Concepção, desenvolvimento e construção de escola secundária. Departamento de Arquitectura, Lisboa. Disponivel em: https://www.academia.edu/27959831/BIM_PROJECT_EXEC UTION PLAN BEP -

CONCEP $\% \bar{C} 3 \% 8 \overline{7} \%$ C $3 \% 830$ DESENVOLVIMENTO E C ONSTRU\%C3\%87\%C3\%830_DE_ESCOLA_SECUND\% $\overline{\mathrm{C}} 3$ $\% 81 R I A$.

Sacks, R.; Eastman, C.; Ghang, L.; Teicholz, P. BIM handbook: a guide to Building Information Modeling for owners, designeers, contractors, and facility mnagers. Third edition. Hoboken, New Jersey: Wiley, 2018.

Succar, B. (2008) Building information modeling framework: a research and delivery foundation for industry stakeholders. Automation in Construction. Disponível em: \&lt;www.elsevier.com/locate/autcon\&gt;.

Succar, B. (2018) BIM adoption policies insights from across the World. ÉTS, Montreal. Disponível em: \&lt;http://gridd.etsmtl.ca/publications/atelier-bimcompetitiviteconstruction-2018/Succar\%20\%20Macro\%20BIM\%20Adoption.pdf\&gt; 